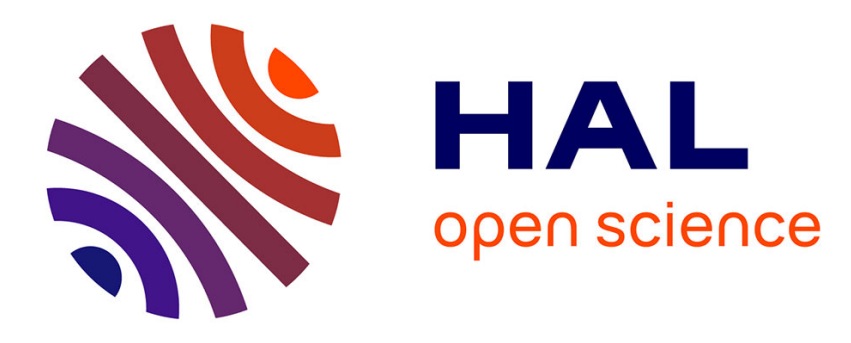

\title{
Multiple-Source Time-Reversal Transmissions in Random Media
}

Andrea Cozza, Florian Monsef

\section{To cite this version:}

Andrea Cozza, Florian Monsef. Multiple-Source Time-Reversal Transmissions in Random Media. IEEE Transactions on Antennas and Propagation, 2014, 62 (8), pp.4269-4281. 10.1109/TAP.2014.2325403 . hal-00990688

\section{HAL Id: hal-00990688 \\ https://hal-centralesupelec.archives-ouvertes.fr/hal-00990688}

Submitted on 13 May 2014

HAL is a multi-disciplinary open access archive for the deposit and dissemination of scientific research documents, whether they are published or not. The documents may come from teaching and research institutions in France or abroad, or from public or private research centers.
L'archive ouverte pluridisciplinaire HAL, est destinée au dépôt et à la diffusion de documents scientifiques de niveau recherche, publiés ou non, émanant des établissements d'enseignement et de recherche français ou étrangers, des laboratoires publics ou privés. 


\title{
Multiple-Source Time-Reversal Transmissions in Random Media
}

\author{
Andrea Cozza, Senior Member, IEEE and Florian Monsef, Member, IEEE
}

\begin{abstract}
The ability of time-reversed signals in reproducing a given time-dependence of the electromagnetic field within random media is investigated. A general setup consisting of multiple sources cooperating in providing the best transmission is considered, where the constructive interference of their individual contributions is meant to improve the quality of the field generation with respect to a single-source setup. The medium response is described by means of tools from random-process theory, for the case of stationary media complex enough to ensure a large number of multi-path contributions. It is shown that even a very weak spatial coherence in the medium is sufficient to significantly hinder the improvement expected from the use of multiple-source scenarios. Experimental results obtained in a reverberation chamber support the validity of the proposed theory. Direct applications of these results can be found in recent proposals about the potential benefits of time-reversed signals used in wireless communications, imaging techniques, as well as in pulsed-field generation devices based on energy compression through dispersive media.
\end{abstract}

Index Terms-Time-reversal transmissions, spatial correlation, complex media, multi-path propagation, statistical electromagnetics.

\section{INTRODUCTION}

Time-reversal transmissions were first introduced in acoustics [1], [2] and initial applications dealt mainly with open media with local inhomogeneities leading to relatively weak propagation aberrations with respect to free-space configurations. In the case of free-space configurations time-reversal can be interpreted as a direct application of Huygens' principle [3]: as well known from the equivalence theorem [4], sampling over a closed surface the field distribution generated by a radiating source allows defining equivalent currents that can recreate on their own a wavefront identical to the one originally generated by the source, be it outward or inward propagating, according to the sign of the time variable. In this respect, time reversal is predated by earlier work in nonlinear optics, particularly on phase-conjugation techniques [5], reminiscent of the ideas behind retrodirective (also known as Van Atta) arrays [6].

For the large family of quasi free-space (or weakly echoic) configurations, time-reversed wavefronts will therefore be a close (time-reversed) replica of the propagative part of the original wavefront, as long as the spatial sampling criterium [7] is fulfilled, by using a sufficient number of field transducers/sources [1], [8]. Conversely, in the case of more complex media, e.g., multiple-scattering media [9]-[14], dominated

A. Cozza and F. Monsef are with the Département de Recherche en Électromagnétisme, Laboratoire des Signaux et Systèmes (L2S), UMR 8506 SUPELEC - Univ Paris-Sud - CNRS, 3 rue Joliot-Curie, 91192 Gif-sur-Yvette, France.

Contact e-mail: andrea.cozza@supelec.fr by discrete scattering phenomena (e.g., collections of small scatterers [15], [16], multi-path environments [17]) or spatially distributed ones (e.g., turbulent media [16], inhomogeneous soils [18]), where field propagation can be modeled by means of random-process tools [16], [19], time-reversed wavefronts are affected by incoherent fluctuations [9], [11], [20], [21].

Although the appearance of these fluctuations could put off users of time-reversed signals in complex media, this nuisance is counterbalanced by a major advantage, since it is no longer necessary to satisfy the spatial sampling criterium. As a matter of fact, single-source configurations have been demonstrated to be capable of recreating high-fidelity versions of a wavefront, when operated by time-reversed signals in a complex medium [20], [22]-[26]. In this case the use of more than one emitter is no longer justified on the basis of the equivalence theorem, but rather on the need to mitigate the level of these fluctuations, by averaging them out thanks to spatial/angle/polarization diversity. Previous results about this topic are available in the literature [27]-[30], but are more concerned with the ability to obtain space-time focusing within random media, rather than assessing the quality of signals thus received.

It is the aim of this paper to understand how multiple-source implementations of time-reversed transmissions in complex media allow to control the relative intensity of the fluctuations, depending on the number of emitters and the statistical properties of the propagation medium, in particular spatial and frequency coherence (or correlation). To this end, a general theoretical approach is developed in section III, based on the concept of energy contrast introduced in section II.

The proposed theory confirms that while multiple sources can improve the quality of the transmission, residual spatial coherence in the medium can significantly reduce the overall effectiveness in mitigating the level of fluctuations. A quantitative analysis of the effects of these predictions is discussed in section IV; experimental results validating them are presented in section $\mathrm{V}$. The results shown are intended to serve as quantitative predictive tools in the design of applications based on the transmission of time-reversed signals, such as in wireless communications, non-destructive testing, remotesensing and imaging techniques.

In this respect, two families of setups for the application of time-reversal transmissions (TRTs) can be defined, both involving the need to generate a faithful reproduction of a template signal $p(t)$ at the receiver-end: (a) a scalar component of the electromagnetic field is the quantity of interest or (b) the output signal observed at the electric port of a receiver. Clearly, these two setups can be regarded as belonging to the same class of problem, by considering that the first case implies the use of an ideal receiver generating an output 
signal $b(t)=\boldsymbol{h} \cdot \boldsymbol{E}(\boldsymbol{r}, t)$, where $\boldsymbol{h}$ is the vector effective length of the receiving transducer found at the position $r$. This kind of response translates the use of an ideal non-dispersive elementary dipole, oriented along the electric-field component of interest $\boldsymbol{h} /\|\boldsymbol{h}\|$.

\section{TRT : COHERENT AND INCOHERENT CONTRIBUTIONS}

We consider a set of $N_{A}$ sources, not necessarily identical, found at the positions $\left\{\boldsymbol{r}_{m}\right\}$. Depending on the application, an observer or a receiver is present at the position $\boldsymbol{r}_{o}$; no assumption is made on the reciprocal orientations of the sources and the observer. Input signals $\left\{a_{m}(t)\right\}$ applied to the transmitting antennas and the overall output signal $b(t)$ are chosen to be represented as power waves, in accordance with standard scattering-parameter formalism [31]. Assuming the medium to be linear, the individual contributions $\left\{b_{m}(t)\right\}$ provided by the excitation of the $m$ th source is given by $b_{m}(t)=a_{m}(t) \star h_{m}(t)$, with $\star$ the time-convolution integral operator and $h_{m}(t)$ the impulse response between the $m$ th source and the receiver output.

The goal of transmission systems is typically to ensure the ability to generate at the receiver output a signal as close as possible to a reference signal $p(t)$, hereafter referred to as the template signal, which is typically taken to be a short pulse in time-reversal literature, but it is not necessarily so; no assumption will be made about this point in this paper since in practical settings, particularly for wireless communications, waveforms are typically more complex and not pulse-like.

When dealing with complex media, if the template signal $p(t)$ were directly applied to an emitter, as soon as its time support $T_{p}$ is shorter than the relaxation time of the medium, any received signal would risk being significantly distorted. Time-reversal transmissions act as a generalization of matched filter, by requiring the use of excitation signals

$$
a_{m}(t)=p(t) \star h_{m}(-t) .
$$

This is the standard definition of time-reversed excitation signals, as originally formulated in acoustics [1]. Unlike for matched-filter theory, the reason for this result is not the maximization of the output signal-to-noise ratio for an additive white gaussian noise channel, but rather to produce the strongest focusing of energy around the receiver [27], [29].

It is well-known that applications of time-reversed signals are limited by the assumption of a stationary medium, in order to ensure the best performance with respect to the quality of reproduction of the template signal; please refer to the papers cited in the introduction for more details. Hence, this property will be assumed throughout this paper.

In order to assess the quality of the output signals generated by this kind of procedure, the mathematical analysis presented in this paper is developed within the framework introduced in [23], where each individual contribution $b_{m}(t)$ to the output signal is expressed as

$$
b_{m}(t)=\alpha_{m} p(t)+f_{m}(t),
$$

i.e., as composed of a coherent part $\alpha_{m} p(t)$, with $\alpha_{m} \geq 0$, proportional to the template signal and a residual part $f_{m}(t)$, orthogonal to $p(t)$.
The overall signal $b(t)$ resulting from the superposition of the $N_{A}$ contributions can therefore be written as

$$
b(t)=p(t) \alpha+f(t)=p(t) \sum_{m=1}^{N_{A}} \alpha_{m}+\sum_{m=1}^{N_{A}} f_{m}(t) .
$$

From $(1), b_{m}(t)=h_{m}(t) \star h_{m}(-t) \star p(t)$, so that for $H_{m}(\nu)$ the Fourier transform of the impulse response of the medium, with $\nu$ the frequency variable, it is convenient to introduce the equivalent transfer functions $W_{m}(\nu)=\left|H_{m}(\nu)\right|^{2}$, relating the Fourier spectrum $P(\nu)$ of the template signal to the actual output signals received when using $(1) ; P(\nu)$ will be assumed to have a compact support, centered at the frequency $\nu_{c}$ and covering a bandwidth $B_{T}$. The coherent coefficients $\left\{\alpha_{m}\right\}$ can therefore be computed by projecting $b(t)$ over $p(t)$, i.e.,

$$
\alpha_{m}=\mathcal{E}_{p}^{-1} \int_{B_{T}} W_{m}(\nu)|P(\nu)|^{2} \mathrm{~d} \nu
$$

with $\mathcal{E}_{p}$ the energy of the template signal $p(t)$.

\section{THEORY OF PEAK AND ENERGY CONTRASTS}

Arguably, the most important figures of merit in TRTs are those measuring its ability to generate a received signal dominated by the coherent part $\alpha p(t)$, at the expenses of the residual part $f(t)$. This feature is commonly assessed by measuring the ratio between the peak value of the received signal and the average intensity of the fluctuations [20], [32]. While this kind of definition makes sense as long as $p(t)$ is expected to be a narrow pulse, it is not general enough in electrical engineering, since one could be interested in transmitting more complex signals, e.g., when dealing with wireless communications; it is therefore necessary to apply a different definition, as the one introduced in section III-A, which can be extended to any kind of signals. More importantly, it is simply related to another figure of merit, the energy contrast. This last quantity is shown in section III-B to only depend on the statistical behavior of the medium's Green's function, independently from the specific choice of the template signal $p(t)$ and more importantly from its bandwidth.

\section{A. Peak contrast}

For the sake of simplicity, it will be assumed that $p(t)$ reaches its peak intensity at $t=0$. We can then introduce the following definition of the peak contrast,

$$
\Lambda_{p}=\frac{\alpha^{2} p^{2}(0)}{\max _{t}\left\langle f^{2}(t)\right\rangle}
$$

as a direct measure of the relative contribution of the coherent and incoherent parts; the latter is measured by the average power of the fluctuations, defined by means of an ensembleaverage operator, represented by the brackets. As proven by our derivation, the shape of the template has a non-negligible impact on the performance of time reversal and its role should 
therefore be explicitly accounted for. For this reason we introduce the shape factors $\chi_{p}$ and $\kappa_{2}$, defined as

$$
\begin{aligned}
\chi_{p} & =\frac{|p(0)|^{2}}{\mathcal{E}_{p}} \\
\kappa_{2}^{2} & =\frac{1}{B_{T}} \frac{\left(\int_{B_{T}}|P(\nu)|^{2} \mathrm{~d} \nu\right)^{2}}{\int_{B_{T}}|P(\nu)|^{4} \mathrm{~d} \nu},
\end{aligned}
$$

where Cauchy-Schwarz inequality indicates that $\kappa_{2} \leq 1$; $\chi_{p}$ measures whether the energy of the template signal is distributed in a narrow span of time as a pulse, or rather as a longer signal. It has dimensions of a bandwidth and its definition implies that $\chi_{p} \leq B_{T}$, where the extreme value is reached only for a sinc pulse, corresponding to a constant power spectral density over $B_{T}$.

In a similar manner, we introduce $\chi_{f}$, defined as

$$
\chi_{f}=\frac{\max _{t}\left\langle f^{2}(t)\right\rangle}{\left\langle\mathcal{E}_{f}\right\rangle}
$$

in order to relate the overall energy of the residual fluctuations to their peak average intensity. The use of ensemble averages in (7) is due to the random nature of the residual fluctuations $f(t)$, inherited from the description of the medium Green's functions as random processes. The major difference between (6) and (7) is that the latter is mostly dependent on the medium and not on the template signal.

From (5), (6) and (7)

$$
\left\langle\Lambda_{p}\right\rangle=\langle\Lambda\rangle \frac{\chi_{p}}{\chi_{f}}
$$

where

$$
\Lambda=\frac{\mathcal{E}_{c}}{\mathcal{E}_{f}}=\frac{\alpha^{2} \mathcal{E}_{p}}{\int_{B_{T}}|F(\nu)|^{2} \mathrm{~d} \nu},
$$

is the energy contrast, defined as the ratio of the energy $\mathcal{E}_{c}$ of the coherent portion of the received signal and the energy $\mathcal{E}_{f}$ of the fluctuating residual part; $F(\nu)$ in (9) is the Fourier transform of the overall fluctuating part $f(t)$. The energy contrast, as it will be proven in section III-B, is practically independent from the choice of the template signal, but strongly related to the statistical properties of the medium and the number of sources used. As a result, (8) allows separating the respective contributions of the medium and the sources from the choice of the template signal, with respect to the quality of the received signals. In particular, the impact of the number of sources used in the transmission only appears in $\Lambda$, hence it is sufficient to study this quantity, as done in the next section.

\section{B. Energy contrast}

In order to compute the ensemble average of the energy contrast $\Lambda$, the following approximation of (9) will be applied:

$$
\langle\Lambda\rangle \simeq \frac{\left\langle\mathcal{E}_{c}\right\rangle}{\left\langle\mathcal{E}_{f}\right\rangle}=\frac{\left\langle\alpha^{2}\right\rangle \mathcal{E}_{p}}{\int_{B_{T}}\left\langle|F(\nu)|^{2}\right\rangle \mathrm{d} \nu},
$$

justified by applying the delta method [33] to the definition of $\Lambda$, with respect to the energies of the coherent and fluctuating parts. It is expected to hold with good precision as long as the number of degrees of freedom underlying the field distribution is much larger than one, as shown in [23]. This condition is not conservative, since it is required when time reversal is applied to complex media, as discussed in [20], [32]. The experimental results presented in $\mathrm{V}-\mathrm{B}$ prove that this approximation is very robust, as they are derived frequency by frequency, without requiring the definition of a bandwidth, as initially stated in the introduction.

From (3)

$$
\left\langle\alpha^{2}\right\rangle=\left\langle\left(\sum_{i=1}^{N_{A}} \alpha_{i}\right)^{2}\right\rangle=\sum_{i=1}^{N_{A}}\left\langle\alpha_{i}^{2}\right\rangle+\sum_{i \neq j}\left\langle\alpha_{i} \alpha_{j}\right\rangle,
$$

while

$$
\begin{gathered}
\int_{B_{T}}\left\langle|F(\nu)|^{2}\right\rangle \mathrm{d} \nu=\sum_{i=1}^{N_{A}} \int_{B_{T}}\left\langle\left|F_{i}(\nu)\right|^{2}\right\rangle \mathrm{d} \nu \\
+\sum_{i \neq j} \int_{B_{T}}\left\langle F_{i}(\nu) F_{j}^{*}(\nu)\right\rangle \mathrm{d} \nu .
\end{gathered}
$$

As it will be shown later, the residual correlation existing between mixed terms in (12) should not be neglected, lest we overestimate the actual performance of the transmission system.

In order to compute these four sets of terms, it is necessary to consider the mutual moments $\left\langle W_{i}\left(\nu_{1}\right) W_{j}\left(\nu_{2}\right)\right\rangle$. As argued in the appendix, the functions involved in these moments, though defined for discrete indexes (i.e., the source identifiers) can be interpreted as continuous parametric fields, i.e., $W_{i}(\nu)=W\left(\boldsymbol{r}_{i}, \nu ; \boldsymbol{h}_{i}\right)$, parameterized by the vector effective height of each respective source. While this would mean dealing with a set of $N_{A}$ different fields, it is possible to simplify our derivation by noticing that a common feature of complex media is that they generate depolarized fields, i.e., on average they do not present dominant polarization components [16], [34], independently from the way the medium was first excited; non-line-of-sight propagation channels comply with this kind of description, which is one of the reasons for the use of reverberation chambers as channel emulators [35]. This property leads to a strong simplification in the statistical representation of Green's function. Under this assumption, if the sources are arbitrarily oriented versions of the same antenna, all the parametric fields share the same statistical moments. This property, discussed in the appendix, is instrumental in our derivation, since it allows dropping the effective heights as parameters, i.e.,

$$
\left\langle W_{i}\left(\nu_{1}\right) W_{j}\left(\nu_{2}\right)\right\rangle=\left\langle W\left(\boldsymbol{r}_{i}, \nu_{1}\right) W\left(\boldsymbol{r}_{j}, \nu_{2}\right)\right\rangle .
$$


As a result the moments $\left\langle W_{i}\left(\nu_{1}\right) W_{j}\left(\nu_{2}\right)\right\rangle$ can now be related to the space-frequency coherence function of what will be referred to as the TRT field $W(\boldsymbol{r}, \nu)$. There should be no confusion at this point that even though (13) is no more dependent on the effective heights $\left\{\boldsymbol{h}_{m}\right\}$, there is no such a thing as a single TRT field, but a set of fields, depending on the characteristics of each source. The TRT field $W(\boldsymbol{r}, \nu)$ considered hereafter should therefore be regarded as an auxiliary function that makes sense only when considering its statistical moments, as those in (13).

We need to recall that

$$
\begin{aligned}
& \left\langle W\left(\boldsymbol{r}_{i}, \nu_{1}\right) W\left(\boldsymbol{r}_{j}, \nu_{2}\right)\right\rangle=\left\langle W\left(\boldsymbol{r}_{i}, \nu_{1}\right)\right\rangle\left\langle W\left(\boldsymbol{r}_{j}, \nu_{2}\right)\right\rangle+ \\
& \mu\left(\boldsymbol{r}_{i}, \boldsymbol{r}_{j}, \nu_{1}, \nu_{2}\right) \sqrt{\sigma_{W}^{2}\left(\boldsymbol{r}_{i}, \nu_{1}\right) \sigma_{W}^{2}\left(\boldsymbol{r}_{j}, \nu_{2}\right)},
\end{aligned}
$$

where $\sigma_{W}^{2}(\boldsymbol{r}, \nu)=\left\langle|W(\boldsymbol{r}, \nu)|^{2}\right\rangle-|\langle W(\boldsymbol{r}, \nu)\rangle|^{2}$ is the variance of the TRT field and $\mu\left(\boldsymbol{r}_{i}, \boldsymbol{r}_{j}, \nu_{1}, \nu_{2}\right)$ is its spectral degree of coherence [36].

In practical scenarios involving complex media where the field can be represented as a large collection of plane waves, e.g., those involving multiple-scattering propagation and interactions with rough surfaces, some further properties can be invoked. First, statistical moments of the Green's dyadic functions can be approximated as being independent from the point of observation, in the same way as wide-sense stationarity is assumed for random time-series, but with respect to space rather than time. As a result $\sigma_{W}^{2}(\boldsymbol{r}, \nu) \simeq \sigma_{W}^{2}\left(\boldsymbol{r}_{o}, \nu\right), \forall \boldsymbol{r}$ so that in the following the argument $r$ will be dropped.

Furthermore, by introducing $\nu_{d}=\nu_{1}-\nu_{2}, \nu_{c}=\left(\nu_{1}+\right.$ $\left.\nu_{2}\right) / 2, \boldsymbol{r}_{c}=\left(\boldsymbol{r}_{i}+\boldsymbol{r}_{j}\right) / 2$ and $\Delta r_{i j}=\left\|\boldsymbol{r}_{i}-\boldsymbol{r}_{j}\right\|$, the degree of coherence can be factorized as

$$
\mu\left(\boldsymbol{r}_{i}, \boldsymbol{r}_{j}, \nu_{1}, \nu_{2}\right) \simeq \mu_{\nu}\left(\boldsymbol{r}_{c}, \nu_{d}\right) \mu_{\boldsymbol{r}}\left(\nu_{c}, \Delta r_{i j}\right),
$$

as argued in [36, section 5.3] for quasi-homogeneous random fields. The two resulting functions in (15), $\mu_{\nu}\left(\boldsymbol{r}_{c}, \nu_{d}\right)$ and $\mu_{\boldsymbol{r}}\left(\nu_{c}, \Delta r_{i j}\right)$, separately account for the degree of frequency and spatial coherence, respectively. While this property can be expected to hold over relatively wide regions of the medium when observed at a single frequency at the time, frequency invariance is a reasonable approximation only when required over a limited bandwidth $B_{W}$. We will assume in the rest of this paper that $B_{W} \gg B_{T}$.

The rationale for approximation (15) is provided by the observation that for complex media the degree of coherence falls to negligible levels if one of two conditions is met: a) as soon as $\left|\nu_{2}-\nu_{1}\right|>B_{c}$, with $B_{c}$ the coherence bandwidth of the medium, as defined later on, or; b) when $\Delta r_{i j}>D_{c}$, the coherence distance (or length) of the medium. When $\left|\nu_{2}-\nu_{1}\right| \simeq 0$, the degree of coherence is dominated by the spatial coherence, while the opposite holds when $\Delta r_{i j} \simeq 0$.

Finally, by assuming that the template signal $p(t)$ operates over a frequency range $B_{T} \gg B_{c}$, the frequency degree of coherence will dominate (15), independently of the observer position, by virtue of the stationarity property; this condition is known to be necessary to ensure effective TRTs in complex media [32]. Hence, $\mu_{\nu}\left(\boldsymbol{r}_{c}, \nu_{d}\right)=\mu_{\nu}\left(\nu_{d}\right)$ in the following. The existence of three separate frequency scales is therefore assumed throughout this work, subject to the condition

$$
B_{W} \gg B_{T} \gg B_{c} \text {. }
$$

The term $\left\langle\alpha_{i} \alpha_{j}\right\rangle$ in (11) can now be computed by using (4), (14) and (15)

$$
\begin{aligned}
& \mathcal{E}_{p}^{2}\left\langle\alpha_{i} \alpha_{j}\right\rangle=\iint_{B_{T}}\left|P\left(\nu_{1}\right)\right|^{2}\left|P\left(\nu_{2}\right)\right|^{2} \times \\
& \left\{\sigma_{W}^{2}\left(\nu_{c}\right) \mu_{\nu}\left(\nu_{d}\right) \mu_{\boldsymbol{r}}\left(\nu_{c}, \Delta r_{i j}\right)+\left\langle W\left(\nu_{1}\right)\right\rangle\left\langle W\left(\nu_{2}\right)\right\rangle\right\} \mathrm{d} \nu_{1} \mathrm{~d} \nu_{2} .
\end{aligned}
$$

Switching to $\nu_{c}$ and $\nu_{d}$, the first part of the integral can be written as

$$
\begin{aligned}
& \iint_{B_{T}}\left|P\left(\nu_{1}\right)\right|^{2}\left|P\left(\nu_{2}\right)\right|^{2} \sigma_{W}^{2}\left(\nu_{c}\right) \mu_{\nu}\left(\nu_{d}\right) \mu_{\boldsymbol{r}}\left(\nu_{c}, \Delta r_{i j}\right) \mathrm{d} \nu_{1} \mathrm{~d} \nu_{2}= \\
& \quad \int \sigma_{W}^{2}\left(\nu_{c}\right) \mu_{\boldsymbol{r}}\left(\nu_{c}, \Delta r_{i j}\right) \times \\
& \quad \int\left|P\left(\nu_{c}-\nu_{d} / 2\right)\right|^{2}\left|P\left(\nu_{c}+\nu_{d} / 2\right)\right|^{2} \mu_{\nu}\left(\nu_{d}\right) \mathrm{d} \nu_{d} \mathrm{~d} \nu_{c}
\end{aligned}
$$

where the second integral is significantly different from zero only for $\nu_{d}<B_{c} \ll B_{T}$, resulting into

$$
\begin{aligned}
& \iint_{B_{T}}\left|P\left(\nu_{1}\right)\right|^{2}\left|P\left(\nu_{2}\right)\right|^{2} \sigma_{W}^{2}\left(\nu_{c}\right) \mu_{\nu}\left(\nu_{d}\right) \mu_{\boldsymbol{r}}\left(\nu_{c}, \Delta r_{i j}\right) \mathrm{d} \nu_{1} \mathrm{~d} \nu_{2} \simeq \\
& \sigma_{W}^{2}\left(\nu_{c}\right) B_{c} \int \mu_{\boldsymbol{r}}\left(\nu_{c}, \Delta r_{i j}\right)\left|P\left(\nu_{c}\right)\right|^{4} \mathrm{~d} \nu_{c},
\end{aligned}
$$

having extracted $\sigma_{W}^{2}\left(\nu_{c}\right)$ from the integral because of (16), while

$$
B_{c}=\int \mu_{\nu}\left(\nu_{d}\right) \mathrm{d} \nu_{d}
$$

is the coherence bandwidth of the medium. By introducing the variability $\varsigma_{W}^{2}(\nu)$ of the TRT field

$$
\varsigma_{W}^{2}(\nu)=\sigma_{W}^{2}(\nu) /\langle W(\nu)\rangle^{2}
$$

we obtain

$$
\begin{aligned}
& \left\langle\alpha_{i} \alpha_{j}\right\rangle \simeq\left\langle W\left(\nu_{c}\right)\right\rangle^{2}[1+ \\
& \left.\mathcal{E}_{p}^{-2} B_{c} \varsigma_{W}^{2}\left(\nu_{c}\right) \int_{B_{T}} \mu_{\boldsymbol{r}}\left(\nu_{c}, \Delta r_{i j}\right)\left|P\left(\nu_{c}\right)\right|^{4} \mathrm{~d} \nu_{c}\right] .
\end{aligned}
$$

This expression can be further simplified by noticing that

$$
\mathcal{E}_{p}^{-2} B_{c} \int_{B_{T}} \mu_{\boldsymbol{r}}\left(\nu_{c}, \Delta r_{i j}\right)\left|P\left(\nu_{c}\right)\right|^{4} \mathrm{~d} \nu_{c} \leq \frac{B_{c}}{B_{T} \kappa_{2}^{2}} .
$$

We can therefore conclude that

$$
\left\langle\alpha_{i} \alpha_{j}\right\rangle \simeq\left\langle W\left(\nu_{c}\right)\right\rangle^{2},
$$

as long as $\kappa_{2}^{2} \gg B_{c} / B_{T}$. The computation of $\left\langle\alpha_{i}^{2}\right\rangle$ goes along the same lines, with the difference that now $\Delta r_{i j}=0$. It results into $\left\langle\alpha_{i}^{2}\right\rangle=\left\langle W\left(\nu_{c}\right)\right\rangle^{2}$, so that the average coherent energy is

$$
\left\langle\mathcal{E}_{c}\right\rangle=N_{A}^{2}\left\langle W\left(\nu_{c}\right)\right\rangle^{2},
$$


a result that corresponds to the case of individual coherent contributions $\left\{\alpha_{i}\right\}$ always constructively interfering, implying a unitary positive correlation. In other words, the spatial invariance of the moments of the TRT field translates into individual coherent contributions that asymptotically behave as deterministic rather than random quantities; this result can be ascribed to the property of self-averaging well known in time-reversal applications [32].

From (2) and (4), the term involving mutual moments in (12) is made up of four contributions

$$
\begin{aligned}
& \int_{B_{T}}\left\langle F\left(\boldsymbol{r}_{i}, \nu\right) F^{*}\left(\boldsymbol{r}_{j}, \nu\right)\right\rangle \mathrm{d} \nu= \\
& \int_{B_{T}}\left\langle W\left(\boldsymbol{r}_{i}, \nu\right) W\left(\boldsymbol{r}_{j}, \nu\right)\right\rangle|P(\nu)|^{2} \mathrm{~d} \nu+\int_{B_{T}}\left\langle\alpha_{i} \alpha_{j}\right\rangle|P(\nu)|^{2} \mathrm{~d} \nu \\
& -\int_{B_{T}}\left\langle W\left(\boldsymbol{r}_{i}, \nu\right) \alpha_{j}\right\rangle|P(\nu)|^{2} \mathrm{~d} \nu-\int_{B_{T}}\left\langle W\left(\boldsymbol{r}_{j}, \nu\right) \alpha_{i}\right\rangle|P(\nu)|^{2} \mathrm{~d} \nu .
\end{aligned}
$$

Recalling (14) and (15)

$$
\begin{aligned}
& \int_{B_{T}}\left\langle W\left(\boldsymbol{r}_{i}, \nu\right) W\left(\boldsymbol{r}_{j}, \nu\right)\right\rangle|P(\nu)|^{2} \mathrm{~d} \nu= \\
& \quad \sigma_{W}^{2}\left(\nu_{c}\right) \int_{B_{T}} \mu_{r}\left(\Delta r_{i j}, \nu\right)|P(\nu)|^{2} \mathrm{~d} \nu+\left\langle W\left(\nu_{c}\right)\right\rangle^{2} \mathcal{E}_{p},
\end{aligned}
$$

a result also appearing in the integrand functions in the last two terms in (26)

$$
\begin{gathered}
\left\langle\alpha_{i} W\left(\boldsymbol{r}_{j}, \nu\right)\right\rangle=\mathcal{E}_{p}^{-1} \int_{B_{T}}\left\langle W\left(\boldsymbol{r}_{j}, \nu\right) W\left(\boldsymbol{r}_{i}, \nu^{\prime}\right)\right\rangle\left|P\left(\nu^{\prime}\right)\right|^{2} \mathrm{~d} \nu^{\prime} \\
\simeq \mathcal{E}_{p}^{-1} \sigma_{W}^{2}(\nu) \mu_{r}\left(\Delta r_{i j}, \nu\right) B_{c}|P(\nu)|^{2}+\langle W(\nu)\rangle^{2}
\end{gathered}
$$

having applied the same line of reasoning as in the derivation of (24). Hence,

$$
\begin{aligned}
& \int_{B_{T}}\left\langle\alpha_{i} W\left(\boldsymbol{r}_{j}, \nu\right)\right\rangle|P(\nu)|^{2} \mathrm{~d} \nu= \\
& \quad=\mathcal{E}_{p}^{-1} \sigma_{W}^{2}\left(\nu_{c}\right) B_{c} \int_{B_{T}} \mu_{r}\left(\Delta r_{i j}, \nu_{c}\right)\left|P\left(\nu_{c}\right)\right|^{4} \mathrm{~d} \nu_{c}+\left\langle W\left(\nu_{c}\right)\right\rangle^{2} \mathcal{E}_{p} \\
& \quad \simeq \mathcal{E}_{p}\left\langle W\left(\nu_{c}\right)\right\rangle^{2},
\end{aligned}
$$

as long as $\kappa_{2}^{2} \gg B_{c} / B_{T}$; this result also holds when the indexes $i$ and $j$ are switched. Stitching together these results yields

$\int_{B_{T}}\left\langle F\left(\boldsymbol{r}_{i}, \nu\right) F^{*}\left(\boldsymbol{r}_{j}, \nu\right)\right\rangle \mathrm{d} \nu \simeq \sigma_{W}^{2}\left(\nu_{c}\right) \int_{B_{T}} \mu_{r}\left(\Delta r_{i j}, \nu\right)|P(\nu)|^{2} \mathrm{~d} \nu$.

Applying essentially the same procedure, the remaining term needed is

$$
\int_{B_{T}}\left\langle\left|F\left(\boldsymbol{r}_{i}, \nu\right)\right|^{2}\right\rangle \mathrm{d} \nu \simeq \sigma_{W}^{2}\left(\nu_{c}\right) \mathcal{E}_{p}
$$

Finally, (10) becomes

$$
\langle\Lambda\rangle \simeq \frac{\varsigma_{W}^{-2}\left(\nu_{c}\right) N_{A}}{1+\mathcal{E}_{p}^{-1} N_{A}^{-1} \sum_{i \neq j} \int_{B_{T}} \mu_{r}\left(\Delta r_{i j}, \nu\right)|P(\nu)|^{2} \mathrm{~d} \nu} .
$$

It can be noticed that

$$
\mu_{i j}=\mathcal{E}_{p}^{-1} \int_{B_{T}} \mu_{r}\left(\Delta r_{i j}, \nu\right)|P(\nu)|^{2} \mathrm{~d} \nu
$$

in (32) represents the degree of spatial coherence between the single-source contributions averaged over $B_{T}$, weighted by $|P(\nu)|^{2}$, while the average energy contrast expected for a single-source configuration is

$$
\left\langle\Lambda^{o}\right\rangle=\left.\langle\Lambda\rangle\right|_{N_{A}=1}=\varsigma_{W}^{-2}\left(\nu_{c}\right),
$$

allowing to restate (32) as

$$
\langle\Lambda\rangle \simeq\left\langle\Lambda^{o}\right\rangle D\left(N_{A}, \bar{\mu}_{r}\right),
$$

with

$$
D\left(N_{A}, \bar{\mu}_{r}\right)=\frac{N_{A}}{1+\left(N_{A}-1\right) \bar{\mu}_{r}}
$$

the improvement provided by exploiting spatial/polarization diversity from $N_{A}$ sources, in an arrangement presenting an average spatial coherence $\bar{\mu}_{r}$

$$
\bar{\mu}_{r}=\frac{\sum_{i \neq j} \mu_{i j}}{N_{A}\left(N_{A}-1\right)} .
$$

\section{PERformance AnAlysis}

The derivation of (35) highlights how the average energy contrast obtained by means of TRT depends on two independent contributions: the average single-source contribution, $\left\langle\Lambda^{\circ}\right\rangle$, and a multiplicative factor $D\left(N_{A}, \bar{\mu}_{r}\right)$, hereafter referred to as the diversity factor. In this section the relative roles of these two terms are discussed.

\section{A. Single-source configurations}

The individual contributions $\left\langle\Lambda^{\circ}\right\rangle$ appear only to depend on the statistical variability $\varsigma_{W}^{2}(\nu)$ of the TRT field and therefore on the statistical properties of the Green's function of the medium, and not on the characteristics of the template signal (bandwidth, shape factor $\chi_{p}$ ). The fact that the bandwidth of the template has no impact on $\left\langle\Lambda^{o}\right\rangle$ implies that the energy efficiency of TRT is firmly constrained by the type of medium we have to deal with.

These limitations in the energy performance (energy contrast) do not apply to the peak contrast, which on the contrary yields more easily to design needs. As a matter of fact, the shape factor $\chi_{p}$ introduced in (6) allows to increase the average peak contrast, since Wiener-Khinchin theorem implies that (8) goes like

$$
\left\langle\Lambda_{p}\right\rangle \sim\left\langle\Lambda^{o}\right\rangle \frac{T_{f}}{T_{p}} \sim\left\langle\Lambda^{o}\right\rangle \frac{B_{T}}{B_{c}},
$$

where $T_{p}$ and $T_{f}$ are the effective time-domain supports (e.g., the half-power durations) of the template (coherent part) 


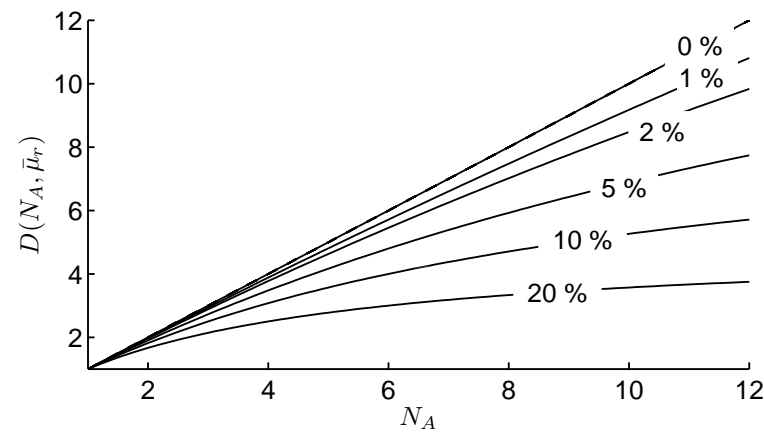

Fig. 1: The diversity factor $D\left(N_{A}, \bar{\mu}_{r}\right)$ as a function of the number of sources $N_{A}$ and the average spatial degree of coherence $\bar{\mu}_{r}$ (in percent units over each line).

and residual fluctuation signals (incoherent part related to the relaxation time of the medium), respectively. In other words, the closer the template to a short pulse, the better the performance of TRTs. The interest of our approach is that it makes clear that while a higher peak contrast can be generated by acting on $B_{T}$ (hence reducing $T_{p}$ ), the overall energy efficiency of TRTs is not under control in complex media; these two quantities act as complementary metrics of the performance of TRTs.

\section{B. Multiple-source configurations}

The function $D\left(N_{A}, \bar{\mu}_{r}\right)$ can have a multiplicative effect on the energy contrast of a single-source configuration, since (36) implies that $D\left(N_{A}, \bar{\mu}_{r}\right) \geq 1$. In the ideal case of $\bar{\mu}_{r}=0$, we expect $D\left(N_{A}, \bar{\mu}_{r}\right)=N_{A}$, resulting in a potentially dramatic improvement of the energy contrast. Since (25) proves that the coherent parts of each individual contribution are hardly distinguishable, the improvement of the energy contrast should not be interpreted as solely due to an increase in the overall coherent transmission. The reasons for the improvement in the overall energy contrast is rather to be found in the perfect decorrelation between the residual fluctuations contributed by each source. Under these circumstances, the average energy of the incoherent fluctuations will sum up as a linear function of $N_{A}$, to be compared with the $N_{A}^{2}$ increase expected for the coherent part.

In the more realistic case of $\bar{\mu}_{r}>0$, the trend of $D\left(N_{A}, \bar{\mu}_{r}\right)$ as a function of $N_{A}$ and $\bar{\mu}_{r}$ is show in Fig. 1. It can be observed that as the number of sources increases, the incremental improvement in the overall energy contrast decreases with $N_{A}$. Moreover, as $N_{A} \rightarrow \infty$, there exists a maximum attainable improvement given by

$$
\lim _{N_{A} \rightarrow \infty} D\left(N_{A}, \bar{\mu}_{r}\right)=\frac{1}{\bar{\mu}_{r}} .
$$

Even a relatively weak average spatial coherence leads to an upper limit to the improvement: e.g., $\bar{\mu}_{r}=0.1$, usually regarded as a negligible level of coherence, directly leads to a maximum improvement of a factor 10 which, though not negligible, implies that it is not possible to do better than the performance that would be obtained with 10 independent

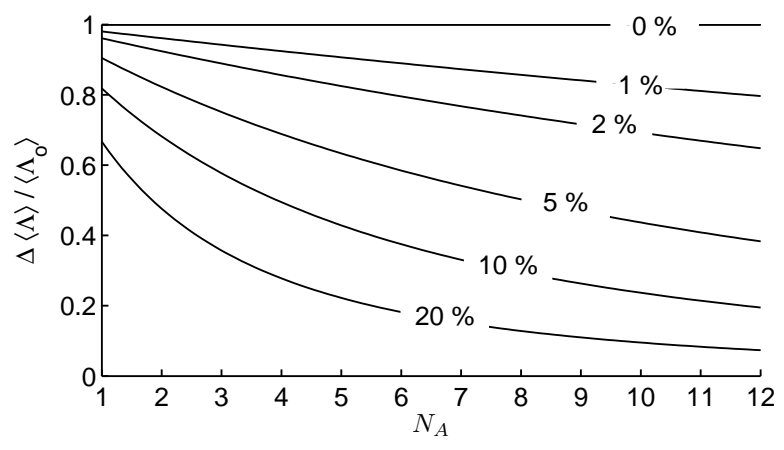

Fig. 2: Incremental contribution to the average overall energy contrast $\langle\Lambda\rangle$ from a source added to $N_{A}$ pre-existing sources, as a function of the average spatial degree of coherence $\bar{\mu}_{r}$ (in percent units over each line).

sources in an idealized setup. Hence, $D\left(N_{A}, \bar{\mu}_{r}\right)$ can be interpreted as an equivalent number of uncorrelated sources taking part to the transmission.

By far worse is the fact that (39) requires an infinite number of real sources excited at the same time, thus implying a huge redundancy level. The limited contribution of each individual source is better observed by computing the incremental improvement due to a single source added to $N_{A}$ already existing, normalized to that of a single source, i.e.,

$$
\frac{\Delta\langle\Lambda\rangle}{\left\langle\Lambda^{o}\right\rangle}=D\left(N_{A}+1, \bar{\mu}_{r}\right)-D\left(N_{A}, \bar{\mu}_{r}\right),
$$

hence

$$
\frac{\Delta\langle\Lambda\rangle}{\left\langle\Lambda^{o}\right\rangle}=\frac{1-\bar{\mu}_{r}}{1-\bar{\mu}_{r}+N_{A} \bar{\mu}_{r}\left(2-\bar{\mu}_{r}\right)+N_{A}^{2} \bar{\mu}_{r}^{2}} .
$$

Since (41) goes like $O\left(N_{A}^{-2}\right)$, it appears that the realized improvement in the performance of TRT after adding a new source decreases relatively fast as $N_{A}$ increases, even for rather low values of $\bar{\mu}_{r}$, as shown in Fig. 2.

It is important to stress the fact that spatial coherence has no link with the more common concept of antenna coupling. Spatial coherence rather implies that the random TRT between one source and the receiver/observer and those of other sources are not entirely uncorrelated random processes. Moreover, spatial coherence is a property that is independent of the structure of the sources and rather depends on the complexity of the medium.

In the design of TRT schemes, it is therefore important to acknowledge this loss of effectiveness of the sources. As a result, there exists a maximum number of sources $N_{A}^{*}$ such that each relative contribution to the overall contrast is higher than a given value $s<1$. Fig. 3 show how $N_{A}^{*}$ evolves with $\bar{\mu}_{r}$ and $s$. Notice how for a requirement of a loss of less than $10 \%$ in the incremental improvement, i.e., $s \geq 90 \%$, it is worthwhile to switch from 1 to 2 sources only if $\bar{\mu}_{r} \leq 5 \%$, a very low level of residual correlation/coherence.

In the case of $\bar{\mu}_{r} \ll 1$ the results in Fig. 3 are well approximated by

$$
N_{A}^{*} \simeq 1+\frac{s^{-1 / 2}-1}{\bar{\mu}_{r}} .
$$




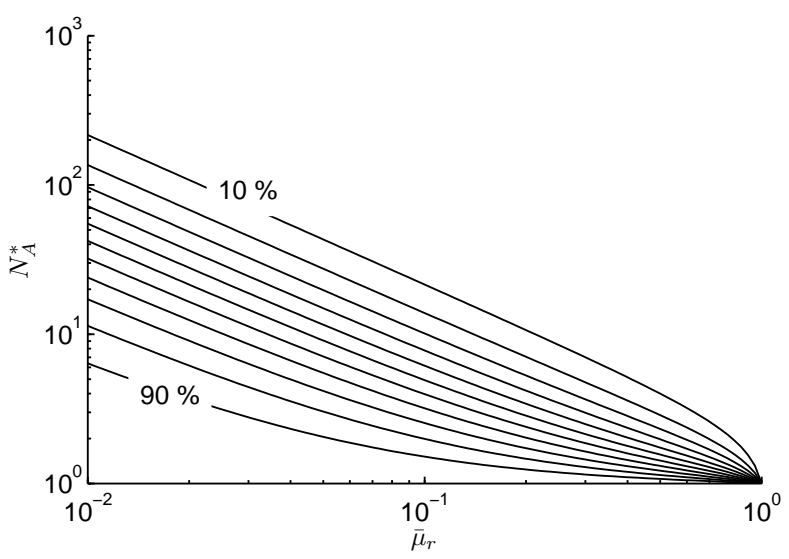

Fig. 3: The maximum number of sources allowed in order to ensure that all of them provide a contribution to the overall contrast at least equal to $s\left\langle\Lambda^{o}\right\rangle$. The 9 curves correspond to values of $s$ going from $10 \%$ to $90 \%$, with a $10 \%$ step.

\section{EXPERIMENTAL VALIDATION}

In order to assess the validity of (35) we chose a reverberation chamber as test case. The rationale for such a choice is threefold: 1) reverberation chambers are routinely used to emulate the effects of wave propagation in complex media of practical interest, such as indoor and outdoor environments, e.g., for hand-held mobile set testing [35], [37], [38]. In this respect, they provide a natural setting for testing our theory; 2) reverberation chambers often come with mechanical field stirrers, intended to modify the boundary conditions for waves propagating through them. A stirrer comes in handy here, as a simple way of generating a large number of random configurations, i.e., an ensemble of media sharing the same statistical properties; 3) it was found in [39] that the variability of the field within a reverberation chamber is far from being a constant value, and can be expected to take rapidly changing values in the lower-frequency range. This property is here interesting as it allows testing our predictions against different values of $\varsigma_{W}^{2}$, i.e., varying random-field statistics, depending on the central frequency of operation; by the same token, spatial coherence is also expected to be a function of frequency [40], so that our predictions can be tested against a whole range of values of $\bar{\mu}_{r}$.

The effectiveness of TRTs is better appreciated in the time domain. In order to simplify the experimental validations, we rather carried out the measurements in the frequency domain; time-domain signals were then post-processed by means of discrete Fourier transforms. Transfer functions were measured with a vector network analyzer, as discussed in section V-A.

Mechanical stirring can only affect that portion of the field (and therefore of the transfer functions) that interacts with it, i.e., submitted to multiple scattering interactions. As a result, line-of-sight propagation between the sources and the receiver position appear as a deterministic contribution shared by each realization of $\left\{H_{m}(\nu)\right\}$ [35]. In order to ensure realizations as independent as possible, line-of-sight contributions were subtracted from the original transfer functions, defining a new

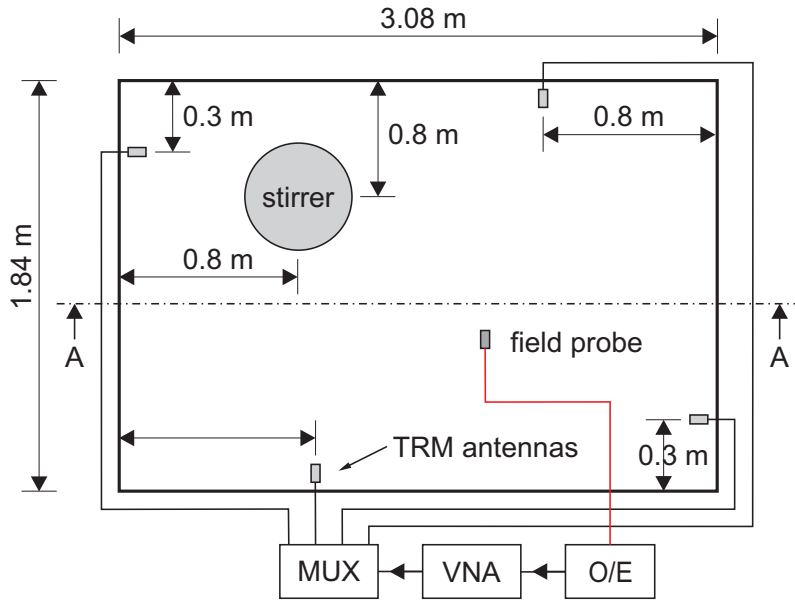

(a)

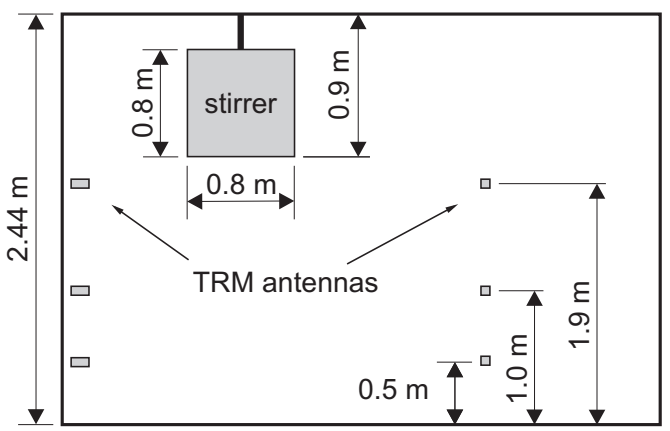

(b)

Fig. 4: The experimental setup used for the validation of the proposed theory. A total of 12 transmitting antennas (see Fig. 5(a)) were mounted over the 4 walls of a reverberation chamber, equipped with a field stirrer (paddle): (a) top view; (b) front view from the AA cut.

set of results as $\left\{H_{m}(\nu)-\left\langle H_{m}(\nu)\right\rangle\right\}$. It is important to stress that this procedure is in no way related to the assumptions invoked throughout our derivation, but is merely a matter of ensuring a set of independent realizations. At the same time this procedure also removes eventual couplings appearing through the excitation circuit.

\section{A. Experimental setup}

A schematic description of the chamber is given in Fig. 4 . A total of 12 sources were included into the chamber; the sources were identical and consisted of printed bow-tie antennas perpendicularly mounted over the walls of the chamber, as shown in Fig. 5(a). Their input ports were connected to a multiplexer through coaxial cables. These antennas where chosen in order to cover the frequency range from 0.5 to 2.0 $\mathrm{GHz}$.

The receiver was an electro-optical probe (Enprobe's EFS105) positioned at the center of the chamber, resting on a 1 $\mathrm{m}$ high styrofoam column. The probe, shown in Fig. 5(b), has 

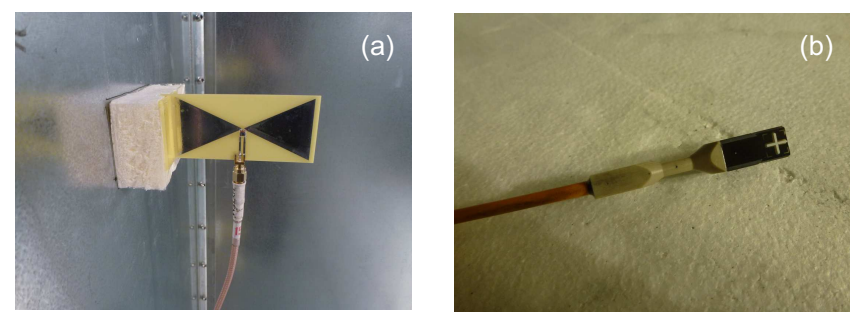

Fig. 5: Experimental setup: (a) one of the 12 bow-tie transmitting antennas, mounted over the walls of the reverberation chamber, kept $3 \mathrm{~cm}$ away from them by a piece of styrofoam; (b) Enprobe's EFS105 optical probe, measuring head $6.6 \mathrm{~mm}$ wide, connected to a optical-fiber cable.

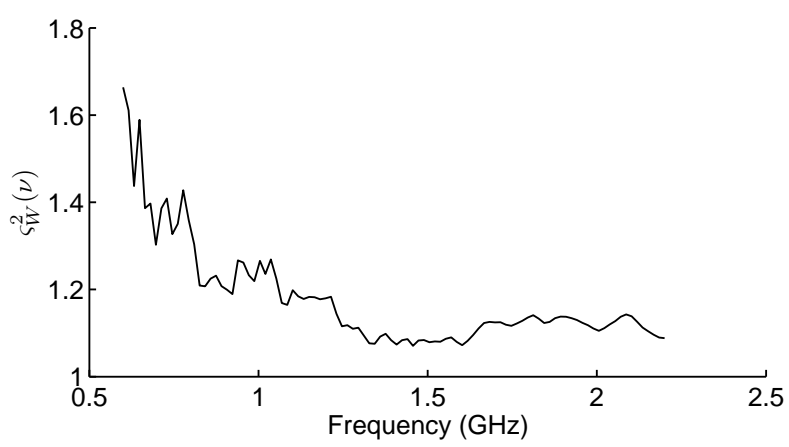

Fig. 6: Estimate of $\varsigma_{W}^{2}$, averaged over the results obtained with 12 sources, smoothed over a $5 \%$ bandwidth.

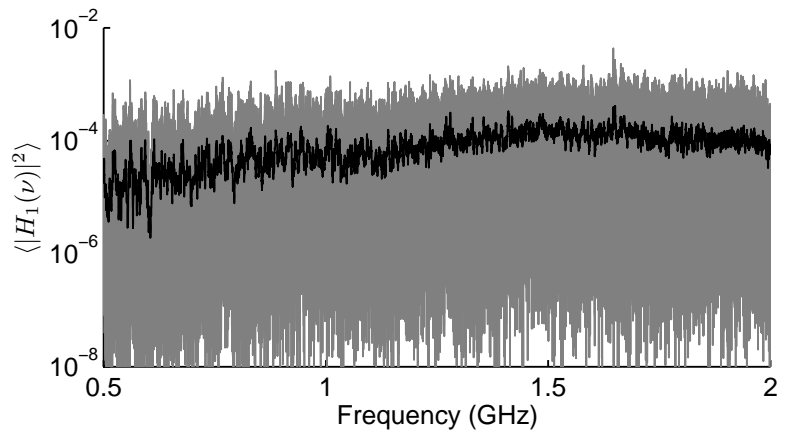

Fig. 7: Plots of the 50 realizations of $W_{1}(\nu)=\left|H_{1}(\nu)\right|^{2}$ (gray) and of their ensemble average $\left\langle W_{1}(\nu)\right\rangle$ (black curve). Fast fluctuations on a short range in the $\left\{W_{1}(\nu)\right\}$ are a consequence of the narrow coherence bandwidth of the medium, as opposed to the smoother evolution of $\left\langle W_{1}(\nu)\right\rangle$, a direct indication of the existence of two separate scales of frequency-related phenomena. the particularity of presenting a negligible scattering crosssection, while behaving as an ideal Hertzian dipole. Its ability to measure phase-shift angles makes it ideally suited for timedomain post-processing. This configuration corresponds to the case where the sources are employed in the generation of a field at the probe position. The probe was operated as a receiver by connecting it to a vector network analyzer (Rohde \& Schwarz ZVB8) through an optic-to-electric converter, while the sources were independently excited one-by-one by means of a multiplexer, as shown in Fig. 4. In this way 12 transfer functions were collected for each realization, corresponding to a single stirrer position. The stirrer consisted of four tilted paddles mounted around a vertical axis; 50 angular positions were considered, in order to generate a corresponding number of sets of transfer functions between the sources and the observation point.

Preliminary results from this setup are given in Fig. 6, where an estimate of the variability of the TRT is shown. This quantity spans values going from 1.1 to 1.7 ; the lower value is close to what is expected for an ideal diffuse-field configuration, related to Rayleigh fading in wireless communications [17], [41], while the higher value is a measure that field propagation is less complex than expected, with a reduced number of degrees of freedom [39]. Moreover, $\varsigma_{W}^{2} \neq 1$ implies that the underlying field cannot be described as a Gaussian process, and therefore neither as a Rayleigh propagation. This fact is important in proving that the agreement between results discussed in the next section and the proposed theory is not limited to simplified configurations (e.g., Rayleigh propagation), but also in intermediary cases closer to practical settings.

Complementary information is provided by the power density results shown in Fig. 7 as a black line, estimated from the 50 realizations of the first of the 12 sources. The received fields appear as essentially behaving as random processes, weakly dependent on the frequency of observation (frequency stationarity). These results are of interest since they imply that condition (16) is satisfied: as a matter of fact, the average power density (black curve) evolves rather smoothly along the frequency axis, while the fast random fluctuations of each realization provide a direct assessment of the frequency coherence of the medium, which is of the order of $100 \mathrm{kHz}$. Furthermore, Fig. 7 does not give away any clue about the fact that the statistics of the medium is fundamentally different at the two ends of the frequency axis, as opposed to Fig. 6. The importance of the information derived from the variability, already exposed in section III-B, will be confirmed in the next section.

\section{B. Output field statistics}

Throughout this section we will consider a gaussian template signal $p(t)$, with a $-20 \mathrm{~dB}$ bandwidth $B_{T}$ modulating a harmonic carrier at the frequency $\nu_{c}$; the values of $B_{T}$ were chosen in order to be much larger than the coherence bandwidth $B_{c}$, as required in the derivation of our theory. The received signals were computed by means of inverse discrete Fourier transforms of the spectra $B_{m}(\nu)=\left|H_{m}(\nu)\right|^{2} P(\nu)$; 

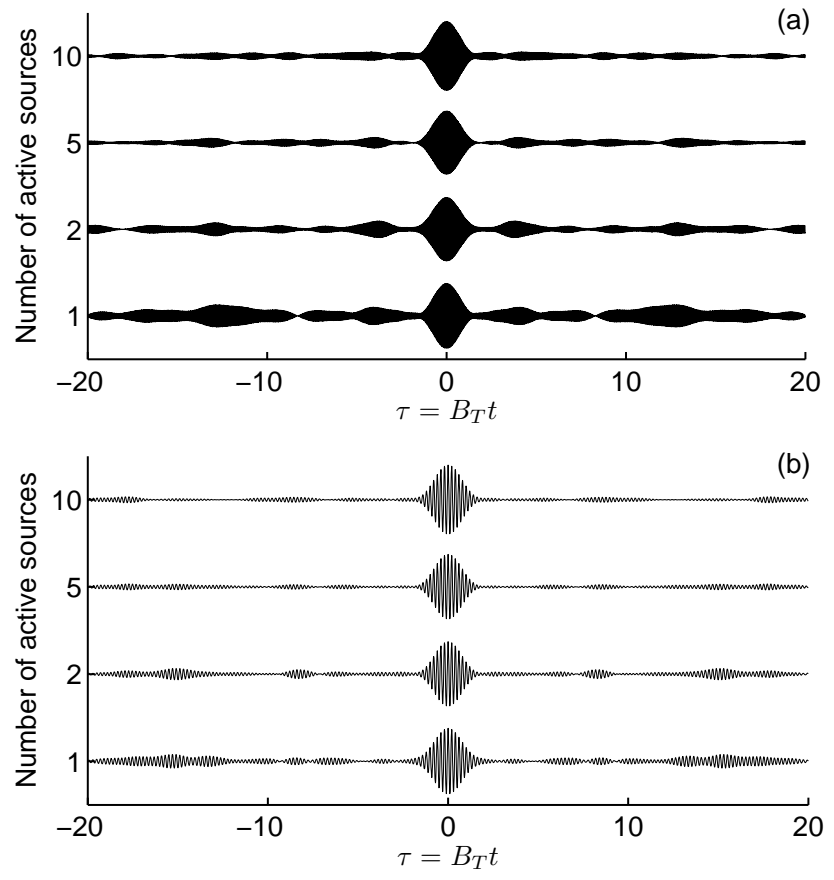

Fig. 8: A single realization of signals received for an increasing number of active sources, for $\nu_{c}=0.7 \mathrm{GHz}$ and: (a) $B_{T} / \nu_{c}=$ $5 \%$; (b) $B_{T} / \nu_{c}=20 \%$. The peak values attained by the signals are practically independent of the number of sources involved.

overall signals were then computed by summing over a chosen subset of individual contributions. For the sake of simplicity, the following convention will be used: for a configuration with $N_{A}$ active sources, the resulting signals correspond to the sum of the first $N_{A}$ sources, without considering all the available combinations. In order to allow a direct comparison of signal amplitude in different configurations, the output signals are always normalized to $N_{A}$, while $p(t)$ has unit energy.

Examples of these signals are given in Fig. 8, for $\nu_{c}=$ $0.7 \mathrm{GHz}$ and two relative bandwidths $B_{T} / \nu_{c}$, namely $5 \%$ and $20 \%$, when exciting an increasing number of sources. While increasing $B_{T}$ leads to a clear improvement in the peak contrast, as already well documented in the available literature about TRT, a further important information is that the resulting fields are practically independent of the number of sources involved. This observation confirms the results obtained in (25), stating that the peak-value of the coherent part is proportional to $N_{A}$, i.e., the individual contributions are practically identical. Still, Fig. 8 also shows that the ratio between the peak coherent value and the background fluctuations does not improve as fast when $N_{A}$ increases; in other words, while the peak received signal increases, its quality is not improving as fast as would be expected for ideally incoherent fluctuations. This behavior is better understood by switching from the intuitive peak contrast to the energy contrast, as argued in section III.

In this respect, we needed to compute the empirical probability density functions (epdf) obtained for the single-source
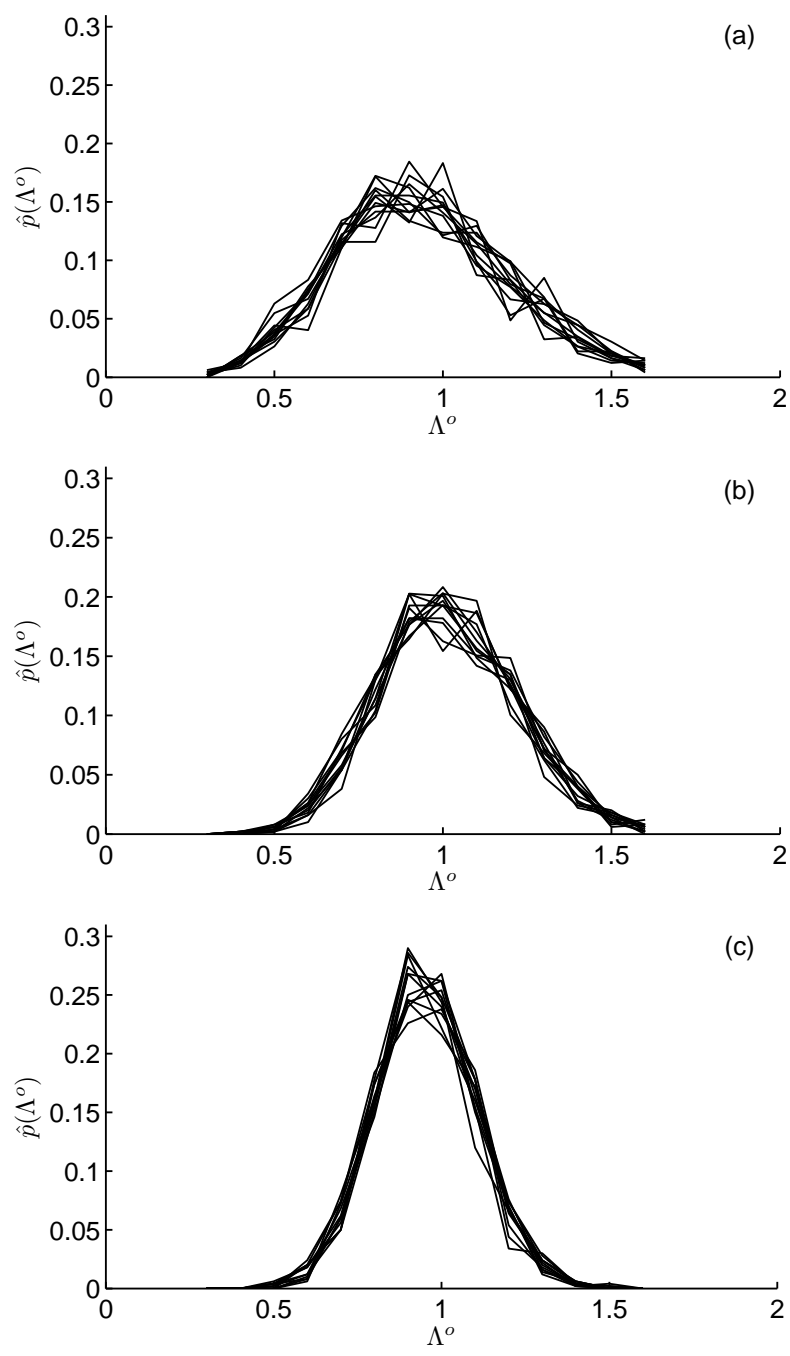

Fig. 9: Empirical probability density functions for $\Lambda^{\circ}$ for three values of the central frequency $\nu_{c}$ : (a) $0.7 \mathrm{GHz}$; (b) $1.3 \mathrm{GHz}$ and (c) $2.0 \mathrm{GHz}$, for a $5 \%$ relative bandwidth. Twelve results are shown on each graph, corresponding to the results obtained from the use of each transmitting antenna.

$\Lambda^{\circ}$, shown in Fig.9, derived for the 12 sources and the data collected for the 50 realizations. These results confirm that the epdfs are practically independent of the source, supporting the arguments presented in the appendix.

The main interest in the use of the energy contrast is that its average value $\left\langle\Lambda^{o}(\nu)\right\rangle$ does not depend on the specific choice of $p(t)$, but only on the medium; evidence for this claim is provided in Fig. 10, where $\left\langle\Lambda^{o}(\nu)\right\rangle$ is computed either applying its definition (9), based on output signals, or from (34), based on estimates of medium statistics; a good agreement is found within a few percent points over the entire frequency range and for three different relative bandwidths, while minor disagreements are likely due to the approximation applied in (10). In particular, going from $B_{T} / \nu_{c}=5 \%$ to $20 \%$, e.g., a fourfold increase, does not lead to any improvement in $\left\langle\Lambda^{o}(\nu)\right\rangle$, as predicted from the proposed theory. Of notable interest is the fact that the agreement is extended to the lower 

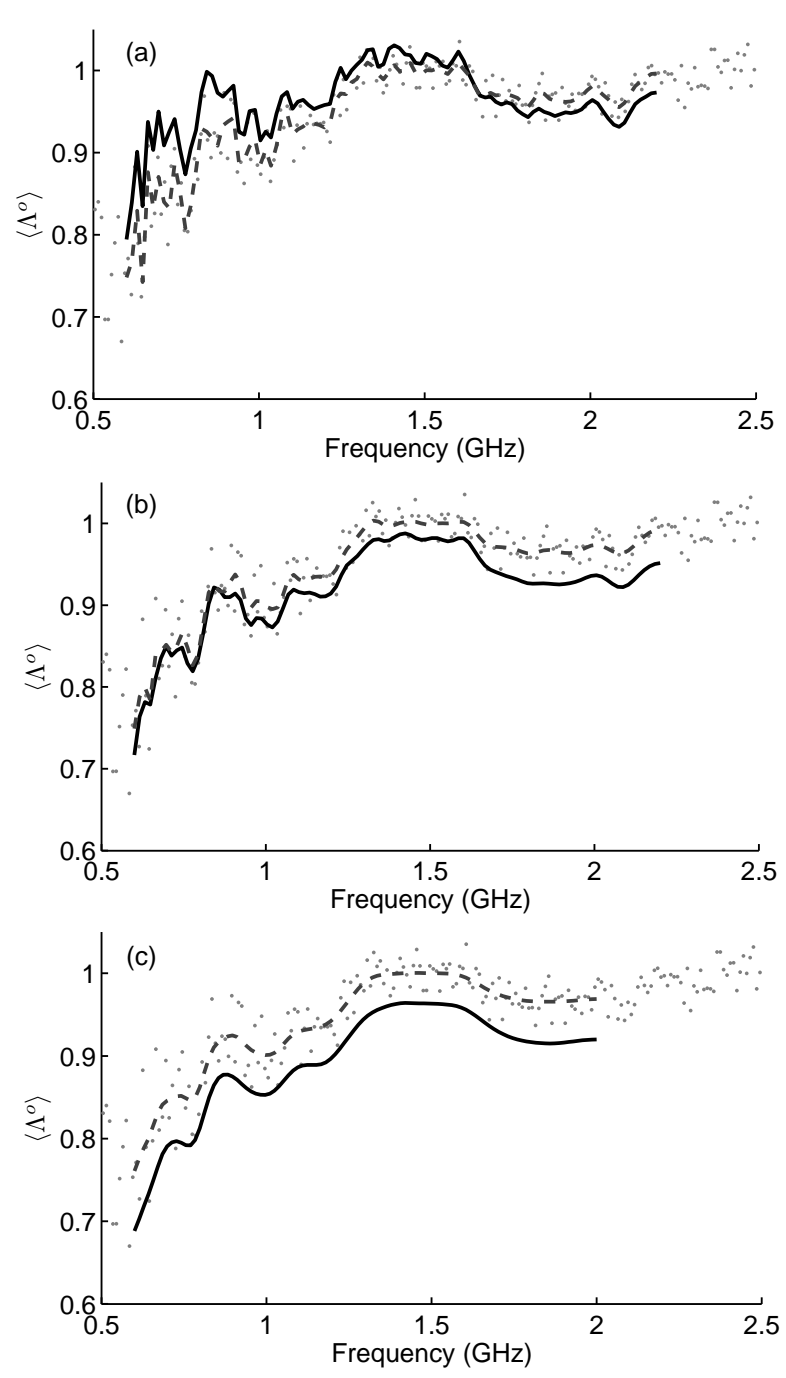

Fig. 10: Comparisons between several estimates of $\left\langle\Lambda^{\circ}\right\rangle$, computed from : the arithmetic average over the 50-realization dataset (solid line); the reciprocal of the variability, i.e., $\varsigma_{W}^{-2}(\nu)$ (gray dots), as predicted by (34); its smoothed version computed by averaging with $|P(\nu)|$ as a weighting function (dashed line). The three graphs relate to three different relative bandwidths: (a) $5 \%$; (b) $10 \%$; (c) $20 \%$.

frequency range, where the field statistics are not compatible with those of a gaussian distribution law, since $\varsigma_{W}^{2} \neq 1$.

Equation (36) states that in order to predict the improvement brought in by the use of multiple-source excitations, the only needed quantity to be computed is $\bar{\mu}_{r}$, according to (37), starting from the residual correlations between the $\left\{W_{m}(\nu)\right\}$ equivalent transfer functions; results are shown in Fig. 11. As expected for a complex medium, the residual spatial correlation is rather low and could be dismissed as negligible.

The predictions given by (36) about the improvement $D\left(N_{A}, \bar{\mu}_{r}\right)$ are compared in Fig. 12 against the measured ratio $\langle\Lambda\rangle /\left\langle\Lambda^{o}\right\rangle$, for three values of $\nu_{c}$ and three relative bandwidths. As predicted, the realized improvement is smaller what would be expected from the deterministic behavior of the individual contributions to the coherent part, i.e.,

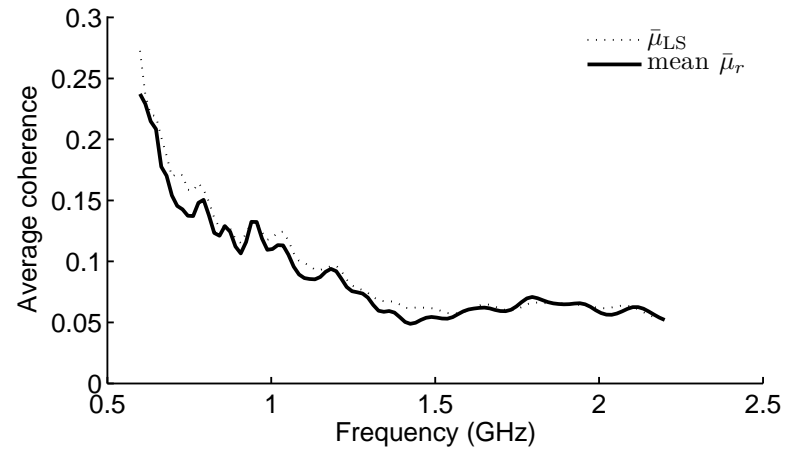

Fig. 11: Estimate of the mean-value of $\bar{\mu}_{r}(\nu)$, computed over the 50 realizations, obtained from the $\left\{W_{m}(\nu)\right\}$ for a $5 \%$ relative bandwidth (solid line), compared to the least-square estimate $\bar{\mu}_{\mathrm{LS}}(\nu)$ derived by fitting the diversity factor to the measured data.

$D\left(N_{A}, \bar{\mu}_{r}\right)=N_{A}$. The reduction in $\langle\Lambda\rangle /\left\langle\Lambda^{o}\right\rangle$ is accurately predicted by (36), based on the $\bar{\mu}_{r}$ estimates in Fig. 11 . The divergence between the theoretical predictions and the experimental results can be explained in terms of residual uncertainties in the estimation of $\bar{\mu}_{r}$ : assuming a $10 \%$ uncertainty provides mutually compatible results.

A more general comparison is possible by computing the least-squares fit of $\langle\Lambda\rangle /\left\langle\Lambda^{o}\right\rangle$, yielding an optimal $\bar{\mu}_{\mathrm{LS}}$, compared in Fig. 11 and Fig. 12 with the estimate of $\bar{\mu}_{r}$. A good agreement is observed over the entire frequency range, supporting the proposed theory as capable of predicting the performance of TRTs in complex media.

\section{CONCLUSIONS}

In this paper we have introduced a theory describing the ability of multiple-source TRT in generating coherent signals in complex media. This theory shows how inevitable spatial correlation puts an upper bound on the improvement brought by the introduction of additional transmitting sources, even for values of coherence that are often regarded as negligible.

The introduction of the energy contrast, as opposed to the more intuitive peak contrast, allows appreciating limitations of the energy efficiency that have been seldom discussed; this topic deserves further research for its practical importance in the optimal design of transmission systems.

Overall, experimental results strongly support the theoretical model presented in section III, and in particular the possibility of factorizing the peak contrast into three separate contributions: a) the ratio between the template bandwidth $B_{T}$ and the coherence bandwidth of the medium; b) the variability of the TRT field supported by the medium; c) the diversity factor $D\left(N_{A}, \bar{\mu}_{r}\right)$, depending on the residual spatial coherence and the number of sources.

These two last points make clear that albeit allowing remarkable performances, time reversal is profoundly affected by physical limitations that cannot be easily compensated by means of signal-processing techniques. 


\section{ACKNOWLEDGEMENTS}

Part of the work here reported was funded by the French National Research Agency through the grant ANR-12-ASTR0005-01, within the framework of the 2012 ASTRID program.

\section{REFERENCES}

[1] M. Fink, "Time reversal of ultrasonic fields. I. Basic principles," IEEE Transactions on Ultrasonics, Ferroelectrics and Frequency Control, vol. 39, no. 5, pp. 555-566, 1992.

[2] M. Fink, D. Cassereau, A. Derode, C. Prada, P. Roux, M. Tanter, J. Thomas, and F. Wu, "Time-reversed acoustics," Reports on Progress in Physics, vol. 63, p. 1933, 2000.

[3] B. Baker and E. Copson, The mathematical theory of Huygens' principle. Chelsea Pub Co, 2003.

[4] R. Harrington, Time-Harmonic Electromagnetic Fields. McGraw-Hill, New York, NY, 1961.

[5] A. Yariv, "Phase conjugate optics and real-time holography," IEEE Journal of Quantum Electronics, vol. 14, no. 9, pp. 650-660, 1978.

[6] E. Sharp and M. Diab, "Van Atta reflector array," IRE Transactions on Antennas and Propagation, vol. 8, no. 4, pp. 436-438, 1960.

[7] E. Joy and D. Paris, "Spatial sampling and filtering in near-field measurements," IEEE Transactions on Antennas and Propagation, vol. 20, no. 3, pp. 253-261, 1972.

[8] M. Fink and C. Prada, "Acoustic time-reversal mirrors," Inverse Problems, vol. 17, p. R1, 2001.

[9] A. Derode, P. Roux, and M. Fink, "Robust acoustic time reversal with high-order multiple scattering," Physical Review Letters, vol. 75, no. 23, pp. 4206-4209, 1995.

[10] P. Roux, B. Roman, and M. Fink, "Time-reversal in an ultrasonic waveguide," Applied Physics Letters, vol. 70, p. 1811, 1997.

[11] C. Draeger, J. Aime, and M. Fink, "One-channel time-reversal in chaotic cavities: Experimental results," The Journal of the Acoustical Society of America, vol. 105, p. 618, 1999.

[12] A. Derode, A. Tourin, J. de Rosny, M. Tanter, S. Yon, and M. Fink, "Taking advantage of multiple scattering to communicate with timereversal antennas," Physical Review Letters, vol. 90, no. 1, p. 14301, 2003.

[13] K. Sarabandi, I. Koh, and M. Casciato, "Demonstration of time reversal methods in a multi-path environment," in IEEE Antennas and Propagation Society International Symposium, vol. 4. IEEE, 2004, pp. 44364439.

[14] I. Vellekoop, A. Lagendijk, and A. Mosk, "Exploiting disorder for perfect focusing," Nature Photonics, vol. 4, no. 5, pp. 320-322, 2010.

[15] H. C. Van De Hulst, Light Scattering by Small Particles. Dover Pubn Inc, 2010.

[16] A. Ishimaru, Wave propagation and scattering in random media. WileyIEEE Press, 1999, vol. 12.

[17] R. Vaughan and J. B. Andersen, Channels, propagation and antennas for mobile communications. Institution of Electrical Engineers, 2003.

[18] M. Yavuz and F. Teixeira, "Full time-domain DORT for ultrawideband electromagnetic fields in dispersive, random inhomogeneous media," IEEE Transactions on Antennas and Propagation, vol. 54, no. 8, pp. 2305-2315, 2006.

[19] R. Bourret, "Stochastically perturbed fields, with applications to wave propagation in random media," Il Nuovo Cimento Series 10, vol. 26, no. 1, pp. 1-31, 1962.

[20] G. Lerosey, J. de Rosny, A. Tourin, A. Derode, G. Montaldo, and M. Fink, "Time reversal of electromagnetic waves," Physical Review Letters, vol. 92, no. 19, p. 193904, 2004.

[21] J. Fouque and G. Papanicolaou, Wave Propagation and Time Reversal in Randomly Layered Media. Springer Verlag, 2007.

[22] S. Kim, W. Kuperman, W. Hodgkiss, H. Song, G. Edelmann, and T. Akal, "Echo-to-reverberation enhancement using a time reversal mirror," The Journal of the Acoustical Society of America, vol. 115, p. $1525,2004$.

[23] A. Cozza, "Statistics of the performance of time reversal in a lossy reverberating medium," Physical Review E, vol. 80, no. 5, p. 56604, 2009.

[24] A. Cozza and A. Abou El Aileh, "Accurate Radiation-Pattern Measurements in a Time-Reversal Electromagnetic Chamber," IEEE Antennas and Propagation Magazine, vol. 52, no. 2, pp. 186-193, 2010.

[25] H. Moussa, A. Cozza, and M. Cauterman, "Experimental demonstration of directive pulsed wavefront generation in reverberation chambers," Electronics Letters, vol. 46, no. 9, pp. 623-624, 2010.
[26] A. Cozza, "Emulating an anechoic environment in a wave-diffusive medium through an extended time-reversal approach," Antennas and Propagation, IEEE Transactions on, vol. 60, no. 8, pp. 3838-3852, 2012.

[27] C. Oestges, A. Kim, G. Papanicolaou, and A. Paulraj, "Characterization of space-time focusing in time-reversed random fields," IEEE Transactions on Antennas and Propagation, vol. 53, no. 1, pp. 283 - 293, 2005.

[28] H. C. Song, W. Hodgkiss, W. Kuperman, W. Higley, K. Raghukumar, T. Akal, and M. Stevenson, "Spatial diversity in passive time reversal communications," The Journal of the Acoustical Society of America, vol. 120, no. 4, pp. 2067-2076, 2006.

[29] J. de Rosny, G. Lerosey, and M. Fink, "Theory of electromagnetic timereversal mirrors," IEEE Transactions on Antennas and Propagation, vol. 58, no. 10, pp. 3139-3149, 2010.

[30] P. Blomgren, P. Kyritsi, A. D. Kim, and G. Papanicolaou, "Spatial focusing and intersymbol interference in multiple-input-single-output time reversal communication systems," IEEE Journal of Oceanic Engineering, vol. 33, no. 3, pp. 341-355, 2008.

[31] K. Kurokawa, "Power waves and the scattering matrix," IEEE Transactions on Microwave Theory and Techniques, vol. 13, no. 2, pp. 194-202, 1965 .

[32] A. Derode, A. Tourin, and M. Fink, "Random multiple scattering of ultrasound. II. Is time reversal a self-averaging process?" Physical Review E, vol. 64, no. 3, p. 36606, 2001.

[33] C. G. Small, Expansions and asymptotics for statistics. CRC Press, 2010.

[34] L. Tsang and J. A. Kong, Scattering of Electromagnetic Waves: Advanced Topics. Wiley-Blackwell, 2001, vol. 26.

[35] C. Holloway, D. Hill, J. Ladbury, P. Wilson, G. Koepke, and J. Coder, "On the use of reverberation chambers to simulate a Rician radio environment for the testing of wireless devices," IEEE Transactions on Antennas and Propagation, vol. 54, no. 11, pp. 3167 -3177, 2006.

[36] E. Wolf, Introduction to the Theory of Coherence and Polarization of Light. Cambridge University Press, 2007.

[37] P. Kildal, "Overview of 6 years R\&D on characterizing wireless devices in Rayleigh fading using reverberation chambers," in International Workshop on Antenna Technology: Small and Smart Antennas Metamaterials and Applications, 2007. IWAT'07. IEEE, 2007, pp. 162-165.

[38] K. Rosengren and P.-S. Kildal, "Radiation efficiency, correlation, diversity gain and capacity of a six-monopole antenna array for a mimo system: theory, simulation and measurement in reverberation chamber,' Microwaves, Antennas and Propagation, IEE Proceedings, vol. 152, no. 1, pp. 7-16, 2005.

[39] A. Cozza, "The Role of Losses in the Definition of the Overmoded Condition for Reverberation Chambers and Their Statistics," IEEE Transactions on Electromagnetic Compatibility, no. 53, pp. 296-307, 2010.

[40] D. Hill and J. Ladbury, "Spatial-correlation functions of fields and energy density in a reverberation chamber," IEEE Transactions on Electromagnetic Compatibility, vol. 44, no. 1, pp. 95-101, 2002.

[41] M. K. Simon and M.-S. Alouini, Digital communication over fading channels. Wiley-Interscience, 2005, vol. 95.

\section{APPENDIX}

The derivation presented in section III-B rests on the idea that while each transmitting antenna generates a different time-reversal field distribution, their statistical moments are independent of the orientation of the sources, as long as they are rotated replicas of the same type of source. In this respect, it is necessary to prove that the first- and secondorder moments of the TRT fields only depend on $\left\{\left\|\boldsymbol{h}_{m}\right\|\right\}$ and are rather dominated by the medium statistics. The main assumption is that the medium generates depolarized fields.

The transfer functions $\left\{H_{m}(\nu)\right\}$ can be related to the vector effective heights of the transmitters and receiver, $\left\{\boldsymbol{h}_{m}\right\}$ and $\boldsymbol{h}_{o}$, respectively. For the sake of simplicity it will be assumed that the transmitters and the receiver are electrically small, whence

$$
H_{m}(\nu)=C(\nu) \boldsymbol{h}_{o}(\nu) \cdot \underline{\boldsymbol{G}}_{\mathrm{ee}}\left(\boldsymbol{r}_{o}, \boldsymbol{r}_{m}, \nu\right) \cdot \boldsymbol{h}_{m}(\nu),
$$


where $\underline{G}_{\mathrm{ee}}$ is the electric-electric Green's dyadic function of the medium, while $C(\nu)$ is a function depending on the reference impedance used in the definition of the scattering parameters; in the following we will set $C(\nu)=1$, with no loss of generality. From (43), we can interpret each transfer function as a continuous parametric field

$$
H_{m}(\nu)=H\left(\boldsymbol{r}_{m}, \nu ; \boldsymbol{h}_{m}\right),
$$

not only defined for the actual position of the $m$ th source, but for a general $\boldsymbol{r}_{m}$.

With no loss of generality, in our derivation it will be assumed that the reference system relating to the observer in $\boldsymbol{r}_{o}$ is defined such that $\boldsymbol{h}_{o}=h_{o} \hat{\boldsymbol{u}}_{k}$, i.e., the observer is aligned to the $k$ th axis $\hat{\boldsymbol{u}}_{k}$. The frequency variable is here dropped for the sake of space. From (43)

$$
W_{m}=\left|\sum_{i, j=1}^{3} h_{o}^{i} h_{m}^{j} G_{e e}^{i j}\left(\boldsymbol{r}_{o}, \boldsymbol{r}_{m}\right)\right|^{2},
$$

where $\left\{G_{e e}^{i j}\right\}$ are the scalar components of the dyadic Green's function. For $\boldsymbol{h}_{o}=h_{o} \hat{\boldsymbol{u}}_{k}$ (45) reduces to

$$
W_{m}=\left|h_{o}\right|^{2}\left|\sum_{i=1}^{3} h_{m}^{i} G_{e e}^{k i}\left(\boldsymbol{r}_{o}, \boldsymbol{r}_{m}\right)\right|^{2},
$$

which, using the convention $X_{i}=G_{e e}^{k i}\left(\boldsymbol{r}_{o}, \boldsymbol{r}_{m}\right)$, leads to

$$
W_{m}=\left|h_{o}\right|^{2} \sum_{i, j} h_{m}^{i} h_{m}^{j *} X_{i} X_{j}^{*}
$$

where $*$ stands for the complex conjugate. The ensemble average $\left\langle W_{m}(\nu)\right\rangle$ is therefore

$$
\left\langle W_{m}\right\rangle=\left|h_{o}\right|^{2} \sum_{i, j} h_{m}^{i} h_{m}^{j *}\left\langle X_{i} X_{j}^{*}\right\rangle .
$$

Following the depolarized-field assumption, $\left\langle G_{e e}^{i j}\left(\boldsymbol{r}_{o}, \boldsymbol{r}_{m}\right) G_{e e}^{p q *}\left(\boldsymbol{r}_{o}, \boldsymbol{r}_{m}\right)\right\rangle=\delta_{i j} \delta_{p q} \sigma_{G}^{2}$, with $\sigma_{G}^{2}$ the variance of each scalar component of the dyadic Green's function; in practical terms, a rotational symmetry or isotropy is introduced in the statistics of the components of the dyadic Green's function. Hence

$$
\left\langle W_{m}\right\rangle=\left|h_{o}\right|^{2}\left\|\boldsymbol{h}_{m}\right\|^{2} \sigma_{G}^{2},
$$

which does not depend on the orientation of the $m$ th source. Field statistics for complex media can be assumed, at least at the first order, as following a complex-valued circular normal distribution, i.e., with zero-mean real and imaginary parts normal distributed and iid, with variances $\sigma_{0}^{2}$, thus $\sigma_{G}^{2}=2 \sigma_{0}^{2}$, quantities directly related to the spectral density $\sigma_{W}^{2}(\boldsymbol{r})$. As argued in section III-B, spatial dependence is here assumed to be negligible at least in the region of space where the sources are found, so that the argument $\boldsymbol{r}$ will be dropped.

In the same way the second-order moment $\left\langle\left|W_{m}\right|^{2}\right\rangle$ can be computed as

$$
\left\langle\left|W_{m}\right|^{2}\right\rangle=\left|h_{o}\right|^{4} \sum_{i, j, p, q} h_{m}^{i} h_{m}^{j *} h_{m}^{p} h_{m}^{q *}\left\langle X_{i} X_{j}^{*} X_{p} X_{q}^{*}\right\rangle .
$$

The assumption of a depolarized field implies that of the four sums above only the terms involving $\{i=j=p=q\},\{i=$ $j \wedge p=q\}$ and $\{i=q \wedge j=p\}$ contribute non-zero moments

$$
\begin{aligned}
& \left\langle\left|W_{m}\right|^{2}\right\rangle\left|h_{o}\right|^{-4}=\sum_{i}\left|h_{m}^{i}\right|^{4}\left\langle\left|X_{i}\right|^{4}\right\rangle+ \\
& 2 \sum_{i \neq j}\left|h_{m}^{i}\right|^{2}\left|h_{m}^{j}\right|^{2}\left\langle\left|X_{i}\right|^{2}\left|X_{j}\right|^{2}\right\rangle,
\end{aligned}
$$

which can be shown to yield

$$
\left\langle\left|W_{m}\right|^{2}\right\rangle=8 \sigma_{0}^{4}\left|h_{o}\right|^{4}\left\|\boldsymbol{h}_{m}\right\|^{4}
$$

and hence

$$
S_{W}=4 \sigma_{0}^{4}\left|h_{o}\right|^{4}\left\|\boldsymbol{h}_{m}\right\|^{4}
$$

The last moment to consider is $\left\langle W_{m}(\nu) W_{n}(\nu)\right\rangle$, which will bring into play the spatial degree of coherence of the medium Green's function. First, it can be shown that

$$
\begin{aligned}
& \left\langle W_{m} W_{n}\right\rangle\left|h_{o}\right|^{-2}=\sum_{i}\left|h_{m}^{i}\right|^{2}\left|h_{n}^{i}\right|^{2}\left\langle\left|X_{i}\left(\boldsymbol{r}_{m}\right)\right|^{2}\left|X_{i}\left(\boldsymbol{r}_{n}\right)\right|^{2}\right\rangle+ \\
& \sum_{i \neq j}\left|h_{m}^{i}\right|^{2}\left|h_{n}^{i}\right|^{2}\left\langle\left|X_{i}\left(\boldsymbol{r}_{m}\right)\right|^{2}\right\rangle\left\langle\left|X_{j}\left(\boldsymbol{r}_{n}\right)\right|^{2}\right\rangle+ \\
& \sum_{i \neq j} h_{m}^{i} h_{n}^{i *} h_{m}^{j} h_{n}^{j *}\left\langle X_{i}\left(\boldsymbol{r}_{m}\right) X_{i}^{*}\left(\boldsymbol{r}_{n}\right)\right\rangle\left\langle X_{j}^{*}\left(\boldsymbol{r}_{m}\right) X_{j}\left(\boldsymbol{r}_{n}\right)\right\rangle .
\end{aligned}
$$

This expression can be simplified by recalling that

$$
\begin{aligned}
\left\langle\left|X_{i}(\boldsymbol{r})\right|^{2}\right\rangle & =2 \sigma_{0}^{2} \forall \boldsymbol{r} \\
\left\langle X_{i}\left(\boldsymbol{r}_{m}\right) X_{i}^{*}\left(\boldsymbol{r}_{n}\right)\right\rangle & =2 \sigma_{0}^{2} \rho_{i}^{\prime}\left(\boldsymbol{r}_{m}, \boldsymbol{r}_{n}\right) \\
\left\langle\left|X_{i}\left(\boldsymbol{r}_{m}\right)\right|^{2}\left|X_{i}\left(\boldsymbol{r}_{n}\right)\right|^{2}\right\rangle & =4 \sigma_{0}^{4}\left[1+\rho_{i}^{\prime \prime}\left(\boldsymbol{r}_{m}, \boldsymbol{r}_{n}\right)\right]
\end{aligned}
$$

where $\rho_{i}^{\prime}\left(\boldsymbol{r}_{m}, \boldsymbol{r}_{n}\right)$ and $\rho_{i}^{\prime \prime}\left(\boldsymbol{r}_{m}, \boldsymbol{r}_{n}\right)$ are the spatial degree of coherence of the $i$ th scalar component of the electric field and its squared modulus, respectively. In order to further simplify our derivation, it is convenient to assume a diffusefield propagation [16]; in this case, by choosing a reference system pertaining to the source part of Green's dyadic function such that the $i$ th axis is either parallel or perpendicular to the direction $\boldsymbol{r}_{m}-\boldsymbol{r}_{n}$, then $\rho_{i}^{\prime \prime}\left(\boldsymbol{r}_{m}, \boldsymbol{r}_{n}\right)=\left[\rho_{i}^{\prime}\left(\boldsymbol{r}_{m}, \boldsymbol{r}_{n}\right)\right]^{2}$, as demonstrated in [40].

This results, after some algebraic manipulations, into

$$
\frac{\left\langle W_{m} W_{n}\right\rangle}{4\left|h_{o}\right|^{4} \sigma_{0}^{4}}=\left\|\boldsymbol{h}_{m}\right\|^{2}\left\|\boldsymbol{h}_{n}\right\|^{2}+\left[\sum_{i=1}^{3} h_{m}^{i} h_{n}^{i *} \rho_{i}^{\prime}\left(\boldsymbol{r}_{m}, \boldsymbol{r}_{n}\right)\right]^{2}
$$

This last expression is actually dependent on the angles between $\boldsymbol{h}_{m}$ and $\boldsymbol{h}_{n}$; depending on the direction cosines of $\boldsymbol{h}_{m}$ and $\boldsymbol{h}_{n}$, the functions $\rho_{i}^{\prime}\left(\boldsymbol{r}_{m}, \boldsymbol{r}_{n}\right)$ will be linearly combined according to different weights. Two special cases can be invoked in order to understand when the final result is not affected by the orientation of the sources.

First, consider the case of identical sources, aligned along a straight line. In this case the direction cosines will be the same independently of the couple $(m, n)$, hence $\left\langle W_{m}(\nu) W_{n}(\nu)\right\rangle$ will only depend on the medium coherence functions. This configuration is often employed in practice, particularly when dealing with linear arrays of sources. 
The second case is that of a more complex geometry, where sources are not necessarily parallel to each other and distributed either along a two- or three-dimensional grid, or randomly. In either case, depending on $(m, n)$, the direction cosines will span any possible value, since even for identical sources their projections over the local reference system defined around the direction $\boldsymbol{r}_{m}-\boldsymbol{r}_{n}$ will change. By modeling the angles as uniformly distributed over all possible directions, the average value of $h_{m}^{i} h_{n}^{i *}$ observed for any $(m, n)$ is expected to be $\left\langle h_{m}^{i} h_{n}^{i *}\right\rangle=\left\|\boldsymbol{r}_{m}\right\|\left\|\boldsymbol{r}_{n}\right\| / 3$, hence (56) will be, according to the law of large numbers, asymptotically equal to

$$
\frac{\left\langle W_{m} W_{n}\right\rangle}{4\left|h_{o}\right|^{4} \sigma_{0}^{4}}=\left\|\boldsymbol{h}_{m}\right\|^{2}\left\|\boldsymbol{h}_{n}\right\|^{2}\left[1+\left(\frac{1}{3} \sum_{i=1}^{3} \rho_{i}^{\prime}\left(\boldsymbol{r}_{m}, \boldsymbol{r}_{n}\right)\right)^{2}\right]_{57},
$$

which only depends on the degree of coherence of the medium.

Recalling the definition of the spatial coherence functions in (15) and making use of (49), (53) and (56) yields

$$
\mu_{r}\left(\Delta r_{m n}\right)=\left[\sum_{i=1}^{3} h_{m}^{i} h_{n}^{i *} \rho_{i}^{\prime}\left(\boldsymbol{r}_{m}, \boldsymbol{r}_{n}\right)\right]^{2}=\left[\frac{\sin \left(k_{o} \Delta r_{m n}\right)}{k_{o} \Delta r_{m n}}\right]_{(58)}^{2}
$$

where the last result holds for the case of a diffusive medium [16], as demonstrated in [40], with $k_{O}=\nu / c_{o}$ the wavenumber and $c_{o}$ the average speed of light through the medium. Equation (58) also proves that $\mu_{m n} \geq 0, \forall m, n$, so that the $\bar{\mu}_{r}$ cannot be smaller than the smallest individual coherences $\left\{\mu_{i j}\right\}$, i.e., they cannot compensate themselves reducing the overall average coherence of the transmission system. 

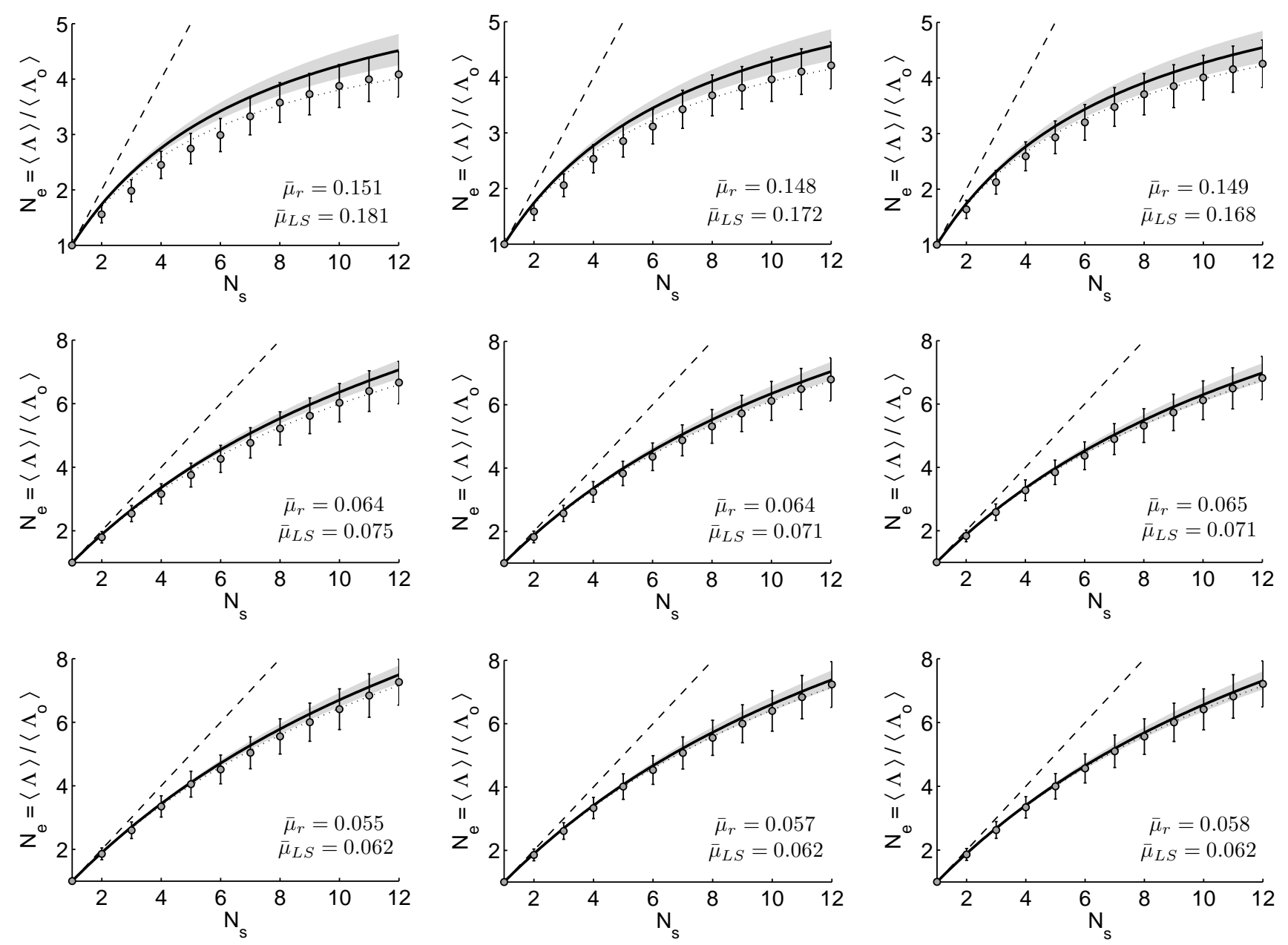

Fig. 12: Equivalent number of independent sources, or diversity factor, as estimated from measurements, for three values of the central frequency $\nu_{c}$ (top to bottom: $0.7,1.3$ and $2.0 \mathrm{GHz}$ ) and three relative bandwidths (left to right: 5, 10 and $20 \%$ ). Each graph presents experimental results as circles, to be compared with the theoretical prediction from (36), shown as a thick solid line, based on the average coherence $\bar{\mu}_{r}$ estimated in Fig. 11. Dotted lines correspond to the use of an optimal average coherence, derived by least-square fitting, while dashed lines are the results that would be expected if the propagation medium presented no spatial coherence. The effects of experimental uncertainties are shown at two levels: 1) a $10 \%$ uncertainty over the estimate of $\bar{\mu}_{r}$, affecting the model prediction, as shown by the shaded area; 2) a $10 \%$ uncertainty over the estimate of $\langle\Lambda\rangle$, as vertical bars. 